\title{
AUTODETERMINACIÓN Y CONTROL GLUCÉMICO EN ADULTOS CON DIABETES TIPO 2
}

\section{SELF-DETERMINATION AND GLYCAEMIC CONTROL IN ADULTS WITH TYPE 2 DIABETES}

Arturo González Jr¹, J. Mercedes Gutiérrez Valverde¹, Milton C. Guevara Valtier', Luis A. Pacheco Pérez² y Geu S. Mendoza Catalán³

${ }^{1}$ Facultad de Enfermería, Universidad Autónoma de Nuevo León. México

2Departamento de Ciencias de la Salud, Campus Cajeme, Universidad de Sonora. México

${ }^{3}$ Facultad de Enfermería, Universidad Autónoma de Baja California. México

\section{Abstract}

Diabetes mellitus is considered a threat to public health worldwide, where community health professionals are key to achieving self-determination and glycemic control. Objective. The purpose of the study was to know the relationship between self-determination and glycemic control in adults with type 2 diabetes. Method. The study design is descriptive correlational, the sample was calculated considering a level of significance of .05 and a power of 90\%, obtaining a total of 204 adults who attended university clinics. Results. $51.4 \%$ of the participants presented glycemic control. No relationship was found between self-determination for diet $\left(r_{s}=.023\right.$; $p=.745)$ and exercise $\left(r_{s}=-.008 ; p=.911\right)$ with glycemic control. It was identified that age was related to external motivation for exercise $\left(r_{s}=.320 ; p=.001\right)$ and for diet $\left(r_{s}=.225 ; p=.001\right)$ and that the years of diagnosis were related to glycemic control $\left(r_{s}=.264 ; p=.001\right)$. Conclusions. Self-determination (internal motivation) is important in the lives of people with DT2, influencing diet and physical exercise, but not directly in glycemic control of people with type 2 diabetes.

Keywords: Self-determination, glycaemic control, adults, type 2 diabetes.

\section{Resumen}

La diabetes mellitus es considerada una amenaza para salud pública a nivel mundial, donde los profesionales de salud comunitaria son claves para lograr la autodeterminación y control glucémico. Objetivo. El propósito del estudio fue conocer la relación entre la autodeterminación y el control glucémico en adultos con diabetes tipo 2. Método. El diseño del estudio es descriptivo correlacional, se calculó la muestra considerando un nivel de significancia de .05 y una potencia del 90\%, obteniéndose un total de 204 adultos que acudieron a clínicas universitarias. Resultados. El $51.4 \%$ de los participantes presentó descontrol glucémico. No se encontró relación entre la autodeterminación para dieta $\left(r_{s}=.023 ; p=.745\right)$ y ejercicio $\left(r_{s}=-.008 ; p=.911\right)$ con el control glucémico. Se identificó que la edad se relacionó con la motivación externa para ejercicio $\left(r_{s}=.320 ; p=.001\right)$ y para dieta $\left(r_{s}=.225 ; p=.001\right)$ y que los años de diagnóstico se relacionaron con el control glucémico $\left(r_{s}=.264 ; \quad p=.001\right)$. Conclusiones. La autodeterminación (motivación interna) es importante en la vida de las personas con DT2, influyendo en la dieta y el ejercicio físico, pero no directamente en el control glucemico de la las personas con diabetes tipo 2 .

Palabras clave: Autodeterminación, control glucémico, adultos, diabetes tipo 2. 
La diabetes mellitus es una enfermedad que afecta al desarrollo de las personas en la sociedad, además de causar sufrimiento en quienes padecen esta enfermedad, repercutiendo en la economía de la familia, comunidades y del país (Organización Mundial de la Salud [OMS], 2016; Federación Internacional de Diabetes [FID], 2017). Entre estas repercusiones económicas y consideradas también como consecuencias de la DT2, está el aumento en el número de consultas médicas y gastos en los sistemas de salud (OMS, 2016).

Así mismo, se calcula que el costo de atención de las personas con diabetes es entre dos y tres veces mayor que quienes no la padecen (Banco Mundial, 2017), afectando a las naciones en la fuerza de trabajo y en condiciones de inequidad y pobreza (Fundación Mexicana para la Salud [FUNSALUD], 2015). De acuerdo con las últimas cifras reportadas en México, la prevalencia de DT2 en este país fue de 10.4\% (OMS, 2016), ocupando el quinto lugar a nivel mundial en prevalencias de diabetes con 12 millones de personas y se estima que para el año 2045 pase a ocupar el cuarto lugar con 21.8 millones (FID, 2017). La DT2 es una enfermedad metabólica y genética que requiere estilos saludables de alimentación, actividad física y tratamiento farmacológico para lograr un control glucémico.

La falta de cumplimiento con las indicaciones de alimentación y ejercicio anteriormente mencionadas en adultos mexicanos puede deberse, en parte, a las barreras percibidas para realizarlas. De acuerdo con la ENSANUT (2016), la falta de motivación es el tercer factor percibido como barrera para realizar ejercicio físico con el 34\% de la población total, mientras que para comer saludablemente funge como el sexto factor con el $28.3 \%$. Aunado a la motivación y considerada como aspecto psicológico se encuentra la autodeterminación, un factor importante que se puede relacionar con la dieta, ejercicio y control glucémico (Gourlan \& Trouilloud, 2015; Halvari et al., 2016; Kálcza-Jánosi, Williams, \& Szamosközi, 2017; Koponen, Simonsen, \& Suominen, 2016; Shahar et al., 2016). La autodeterminación se le define como una característica de la funcionalidad de las personas, que implica la capacidad de elección realizada por sí mismos mediante la experiencia percibida de la motivación interna y externa (Deci \& Ryan, 1985) relacionadas con dieta, ejercicio y control glucémico.
La motivación interna se basa en las decisiones individuales y propias de las personas, ayudando en la elección de estilos de vida y donde factores como las recompensas, los controles interpersonales y las implicaciones del ego afectan tanto la motivación como el interés interno. Por otro lado, la motivación externa tiene que ver con el entorno de la persona, del apoyo exterior como la familia, amigos y sociedad. Ambas motivaciones contribuyen a la autodeterminación, sin embargo, la extrínseca por sí sola no lleva al individuo a la autodeterminación. Se ha identificado que personas con alta autodeterminación logran más una práctica de actividad fisica y se sienten más satisfechos a diferencia de quienes no la presentan (Gourlan \& Trouilloud, 2015). La motivación es un aspecto que se aborda durante el cuidado brindado por enfermería, y que ayuda para animar y empoderar a las personas con DT2 para hacer cambios en su comportamiento buscando la salud personal.

Existe evidencia reportada en países como Estados Unidos, Noruega, Malasia y Rumania de la relación entre la autodeterminación (motivación interna) y el control glucémico (Halvari et al., 2016; Shahar et al., 2016; KálczaJánosi et al., 2017), sin embargo, en México no se ha identificado evidencia que soporte esta relación. Por lo anterior el propósito del presente estudio es conocer la relación entre la autodeterminación y el control glucémico en adultos mexicanos con DT2.

\section{MÉTODO}

\section{Diseño y Participantes}

El diseño del estudio fue descriptivo correlacional de corte transversal. La población se conformó de 555 adultos con diagnóstico de DT2 atendidos en cuatro Clínicas Universitarias de Salud del área metropolitana de Monterrey, Nuevo León (San Rafael, Vicente Guerrero, 21 de enero y Pueblo Nuevo). El muestreo fue no probabilístico por conveniencia, tomando en cuenta los criterios de inclusión (ser mayor de edad, padecer diabetes tipo 2 y contar con 2 horas mínimas de ayuno), con asignación proporcional al tamaño del estrato, donde los estratos fueron las clínicas. Las unidades muestrales se seleccionaron por orden de llegada a cada unidad de salud. La muestra se calculó por medio del software n'QueryAdvisor Versión 7.0, mediante la fórmula: $n=$ 
$\mathrm{NZ}^{2} \mathrm{~S}^{2} / \mathrm{d}^{2}(\mathrm{~N}-1)+\mathrm{Z}^{2} \mathrm{~S}^{2}$, con un nivel de significancia de $.05 \mathrm{y}$ una potencia del $90 \%$, por lo que la muestra se conformó por 204 personas.

\section{Instrumentos}

Se utilizó una encuesta de recolección de datos (cédula de datos personales) que incluyó edad, sexo, años de educación formal, años de diagnóstico, glicemia capilar y tipo de tratamiento farmacológico y no farmacológico (dieta y ejercicio).

Para conocer la autodeterminación fue necesario identificar el tipo de motivación que presentaban los participantes. La motivación se divide en interna y externa, las cuales se midieron utilizando el Cuestionario de Autorregulación del Tratamiento en cuanto a Diabetes (Williams, Freedman \& Deci, 1998), conformada por dos secciones, la primera hace referencia a la razón por la que las personas siguen su dieta; la segunda es la razón por la que hacen ejercicio regularmente. Cada sección incluye dos sub-escalas: regulación autónoma (motivación interna) y regulación controlada (motivación externa).

Los reactivos que evaluaron la autodeterminación (motivación interna) comprendían razones para seguir su dieta y/o hacer ejercicio regularmente, entre las que se destacan: hacerlo para mantenerme saludable, porque creen que es lo correcto y lo mejor para ellos y porque son alternativas que desean llevar a cabo. Por otro lado, los que evaluaron la motivación externa comprendía razones para seguir su dieta y/o hacer ejercicio regularmente como: hacerlo para que alguien más no se molestara con ellos, por vergüenza, hacerlo porque alguien más se los dice, para que alguien más vea que pueden hacerlo, porque su médico se los indica y por culpabilidad si no lo hacen. Este cuestionario cuenta con 22 reactivos en total que incluyen dieta y ejercicio.

Para el análisis estadístico, las puntuaciones se convirtieron en índices, el puntaje crudo esperado oscila entre 0 y 100 puntos, donde el punto de corte fue 50 y los valores más altos (> a 50 puntos) reflejan una mayor motivación interna y/o motivación externa. Cabe destacar que este instrumento no se ha aplicado en población mexicana, no obstante, se realizó traducción y retraducción con especialistas y se sometió a validación de contenido por expertos en el tema, posteriormente se realizó una prueba piloto, y tuvo un alfa de Cronbach de .60
Para determinar el control glucémico se realizaron pruebas de glucosa capilar en ayunas. Se utilizó un glucómetro marca Accu-Chek® Instant, con capacidad de medición dentro de un rango de 10 mg/dL - 600 mg/dL, requiriendo un tamaño de muestra hemática de $.6 \mu \mathrm{L}$, con un tiempo de medición de 5 segundos aproximadamente. La prueba se realizó después de confirmar el ayuno (2 horas mínimo). Para considerar los resultados como control o descontrol glucémico, se apegó a las recomendaciones establecidas por la Asociación Americana de Diabetes (ADA, 2018). De esta forma se consideró control glucémico a cifras de glucosa plasmática capilar prepandial de 80 a $130 \mathrm{mg} / \mathrm{dL}$ y descontrol glucémico a cifras $<80 \mathrm{mg} / \mathrm{dL}$ o $>130 \mathrm{mg} / \mathrm{dL}$ prepandial.

\section{Procedimiento}

Para realizar el presente estudio se solicitó la autorización y registros de los pacientes con DT2 y a los directivos de la institución donde se realizó el estudio, ubicado en el área metropolitana de Monterrey, Nuevo León, México. Posteriormente se realizó la invitación de manera verbal y por medio de tarjetas con nombre del proyecto, día, hora y lugar de atención por parte del investigador.

La recolección de datos se realizó durante los meses de diciembre del 2018 a febrero del 2019. Se les invitó a participar en el estudio, explicando que su participación consistiría en realizar una glicemia capilar con el requisito de 2 horas mínimas de ayuno y contestar una serie de preguntas realizadas por el investigador. Una vez que el participante aceptó ser parte del estudio y firmó el consentimiento, se procedió a realizar la prueba de glicemia capilar, el instrumento fue contestado a lápiz y papel en forma de entrevista y al finalizar se agradeció su participación.

\section{Análisis de datos}

Para el procesamiento y análisis de los datos se utilizó el paquete estadístico Statistical Package for the Social Sciences (SPSS) versión 21 para Windows. Se evaluó primeramente la confiabilidad de los instrumentos a través del coeficiente alfa de Cronbach. Seguido se utilizó estadística descriptiva como frecuencias, porcentajes y medidas de tendencia central y de variabilidad, esto para describir la muestra del estudio y las variables utilizadas. Previo al análisis se procedió a calcular sumatoria, 
promedio e índice del instrumento. Para identificar las relaciones entre las variables del estudio se utilizó el Coeficiente de Correlación de Spearman mediante el Modelo de Regresión Lineal Múltiple.

\section{Consideraciones éticas}

El estudio se apegó a lo estipulado en el reglamento de la Ley General de Salud en Materia de Investigación para la Salud (Secretaría de Salud, 1987) y fue aprobado por el Comité de Investigación, de Ética en Investigación y de Bioseguridad con número de registro: FAEN-M-1456.

\section{RESULTADOS}

De acuerdo con las características sociodemográficas se muestra que la mayoría de los participantes fueron mujeres (72.5\%). El promedio de edad de los participantes fue de 60 años ( $D E=12.3$ ), presentando edades mínimas de 26 y máxima de 86 años. Respecto a la educación formal se identificó una media de educación de 7.9 años $(D E=3.6)$, donde se identificó a participantes sin escolaridad hasta participantes con nivel de educación universitaria (de hasta 16 años de escolaridad). Con relación a los años de diagnóstico, se presentaron participantes con un año hasta 40 años de diagnóstico $(M=9.3 ; D E=8.5)$. Con relación a la prueba bioquímica, la glucemia capilar promedio fue de $163 \mathrm{mg} / \mathrm{dL}(D E=81.3)$, presentando rangos mínimos de 74 hasta $518 \mathrm{mg} / \mathrm{dL}$. Posterior a ello se identificó que un porcentaje amplio de los participantes $(51.5 \%)$ presentó descontrol glucémico (Tabla 1).

Tabla 1. Clasificación de la glicemia capilar en adultos con DT2

\begin{tabular}{lcc}
\hline & $f$ & $\%$ \\
\hline Descontrol glucémico & 105 & 51.5 \\
Control glucémico & 99 & 48.5
\end{tabular}

Nota: $n=204 ; f=F r e c u e n c i a ; \%=P o r c e n t a j e$.

Con el fin de conocer los datos asociados al tratamiento, se encontró que el 100\% de los participantes refirieron tener indicado tratamiento farmacológico. De los cuales refirieron que el $75 \%$ fue hipoglucemiantes orales, el $9.3 \%$ por medio de administración de insulina y el $15.7 \%$ presentaron combinación de tratamiento oral e insulina. Por otra parte, en el seguimiento del tratamiento no farmacológico (alimentación y ejercicio) se identificó que los participantes que tienen recomendaciones específicas de alimentación fueron del 38.2\% y los que tenían recomendaciones para ejercicio fue de $31.9 \%$. En la Tabla 2 se puede observar las recomendaciones específicas que presentaron los participantes.

Tabla 2. Tratamiento no farmacológico

\begin{tabular}{|c|c|c|c|c|c|}
\hline \multicolumn{3}{|c|}{$\begin{array}{c}\text { Recomendaciones de consumo } \\
\text { de alimentos específicos }\end{array}$} & \multicolumn{3}{|c|}{$\begin{array}{l}\text { Recomendaciones de } \\
\text { realización de ejercicio fisico } \\
\text { específicos }\end{array}$} \\
\hline & $f$ & $\%$ & & $f$ & $\%$ \\
\hline $\begin{array}{l}\text { Alimentos } \\
\text { específicos }\end{array}$ & & & $\begin{array}{l}\text { Ejercicio } \\
\text { especifico }\end{array}$ & & \\
\hline Frutas & 31 & 15.2 & Caminar & 63 & 30.9 \\
\hline Verduras & 29 & 14.2 & Bailar & 2 & 1 \\
\hline Pollo & 16 & 7.8 & $\begin{array}{l}\text { Ejercicio que } \\
\text { no puede } \\
\text { realizar }\end{array}$ & & \\
\hline Pescado & 2 & 1 & Correr & 1 & 0.5 \\
\hline \multicolumn{6}{|c|}{$\begin{array}{l}\text { Alimentos que } \\
\text { no puede } \\
\text { consumir }\end{array}$} \\
\hline Harina & 12 & 5.9 & & & \\
\hline Grasas & 10 & 4.9 & & & \\
\hline Azúcar & 6 & 2.9 & & & \\
\hline
\end{tabular}

Respecto a la evaluación de la autodeterminación (motivación interna), se encontró la mayoría de los participantes estaban autodeterminados para realizar ejercicio y dieta saludable (Tabla 3) y que dentro de las razones para seguir su dieta y/o hacer ejercicio regularmente, el $98.5 \%$ era para mantenerme saludable, el $98.5 \%$ porque creían que era lo correcto, el 99\% porque creían que era lo mejor para ellos y el $97.1 \%$ porque son alternativas que desean llevar a cabo. Con relación a la motivación externa, se identificó que dentro de las razones para seguir su dieta y/o hacer ejercicio regularmente, el $15.7 \%$ era para que alguien más no se molestara con ellos, el $43.1 \%$ por vergüenza, el $67.3 \%$ era porque alguien más se los decía, el $43.1 \%$ era para que alguien más viera que podían hacerlo, el $41.7 \%$ era porque su médico se los indicaba y el $93.1 \%$ por culpabilidad si no lo hacían.

Respecto al objetivo de la presente investigación que busca determinar la relación entre la autodeterminación (motivación interna) y el control glucémico, los resultados reflejan que no hubo relación significativa entre la autodeterminación para dieta 
$\left(r_{s}=.023 ; p=.745\right)$ y ejercicio $\left(r_{s}=-.008 ; p=.911\right)$ con el control glucémico (Tabla 4).

Tabla 3. Porcentaje de autodeterminación (motivación interna) para dieta y ejercicio en adultos con DT2

\begin{tabular}{lcccccc}
\hline & \multicolumn{5}{c}{ Autodeterminación } \\
\cline { 2 - 7 } $\begin{array}{l}\text { Puntaje del } \\
\text { índice } \\
\text { estimado }\end{array}$ & \multicolumn{5}{c}{ MID } \\
& \multicolumn{7}{c}{ MIE } & & & \\
& fi & $n i$ & $\%$ & fi & ni & $\%$ \\
$>50$ pts. & 204 & 1 & 100 & 203 & .99 & 99.5 \\
$\leq 50$ pts. & 0 & 0 & 0 & 1 & .004 & .5
\end{tabular}

Nota: $n=204$; fi=Frecuencia absoluta; $n i=F r e c u e n c i a$ relativa; \%=Porcentaje; MID=Motivación interna para dieta; MIE=Motivación interna para ejercicio.

Tabla 4. Coeficiente de Correlación de Spearman para glicemia capilar, autodeterminación (motivación interna) y motivación externa para dieta y ejercicio en adultos con DT2

\begin{tabular}{|c|c|c|c|c|}
\hline & \multicolumn{2}{|c|}{$\begin{array}{l}\text { Autodeterminación } \\
\text { (Motivación interna) }\end{array}$} & \multicolumn{2}{|c|}{ Motivación externa } \\
\hline & Dieta & Ejercicio & Dieta & Ejercicio \\
\hline Glicemia capilar & .023 & -.008 & .027 & .006 \\
\hline
\end{tabular}

Asimismo, como datos adicionales, se encontró relación entre la edad y la motivación externa para ejercicio $\left(r_{s}=.320 ; p=.001\right)$ y relación entre la edad y la motivación externa para dieta $\left(r_{s}=.225 ; p=.001\right)$, donde a mayor edad mayor motivación externa para hacer ejercicio y llevar una dieta, aunque dichas asociaciones fueron débiles a pesar que existen diferencias significativas. También se puede observar que solo los años de diagnóstico se relacionó con el control glucémico $\left(r_{s}=.264 ; p=.001\right)$, donde a más años de diagnóstico mayor control glucémico.

\section{DISCUSSION}

El presente estudio se caracterizó por incluir hombres y mujeres en edad adulta que asistieron a clínicas universitarias de la UANL entre diciembre del 2018 y febrero del 2019, encontrando que la mayoría de la población fueron mujeres. Este hallazgo podría atribuirse a que, en la población mexicana, así como en el estado de Nuevo León, el número de mujeres con DT2 es mayor que el de los hombres (ENSANUT, 2016; Instituto Nacional de Salud Pública, 2013; OMS, 2016). Aunado a esto, en la mayoría de los estudios donde se abordan aspectos de la DT2 incluyen en mayor proporción a participantes femeninos (Aspano et al., 2016; KálczaJánosi et al., 2017; Shahar et al., 2016; Trief et al., 2016). En relación con la edad, el promedio fue 60 años, este dato es similar a lo reportado en los resultados de la ENSANUT (2016), donde se menciona que la mayoría de las personas con DT2 tienen entre 60 y 79 años.

Por otro lado, el promedio de años de educación formal fue de 7.9 años, equivalente a educación secundaria, inferior a la media nacional (9.2 años) y a la media del estado de Nuevo León (10.3 años) reportado por el Instituto Nacional de Estadística y Geografía (INEGI, 2015). Esta diferencia se podría atribuir a la proporción de pobreza en la población de los municipios donde se llevó a cabo el estudio (Guadalupe 16.7\% y Apodaca 13.1\%) de acuerdo con el Consejo Nacional de Evaluación de la Política de Desarrollo Social (CONEVAL, 2015). El promedio del tiempo de diagnóstico fue de 9.3 años, este dato es menor a lo reportado por Gourlan \& Trouillound (2015) y similar al de Kálcza-Jánosi et al., (2017). Esta diferencia podría atribuirse a que el tamaño de muestra del estudio de Gourlan \& Trouillound (2015) es mayor que el considerado para esta investigación.

Una de las características de la muestra estudiada es el alto el porcentaje de participantes que reportaron cifras de glucosa plasmática en descontrol glucémico (51.5\%), a pesar de que la totalidad tenían indicado tratamiento farmacológico, en su mayoría hipoglucemiantes orales (75\%). Posiblemente este resultado podría deberse a la ausencia de recomendaciones específicas de alimentación y ejercicio físico, y al bajo nivel educativo antes mencionado, concordando con los resultados reportados por Halvari et al. (2016), Kálcza-Jánosi et al. (2017) y Shahar et al. (2016).

Por otro lado, aunque se encontró que casi la totalidad de la población estaba autodeterminada tanto para llevar una dieta sana como para hacer ejercicio fisico, la autodeterminación no se relacionó con el control glucémico. Posiblemente aunque las personas con DT2 estudiadas en su mayoría tenían motivaciones internas para realizar ejercicio fisico y llevar una dieta saludable, existen diversos factores que pueden influir en el control y/o descontrol glucémico, como el estrés y la depresión (Sánchez-Cruz, Hipólito-Lóenzo, Mugártegui-Sánchez \& 
Yáñez-González, 2016), y enfermedades bucales (Mazzini, Ubilla \& Moreira, 2017; Sanz et al., 2017; Ternois, 2017; Saengtipbovorn \& Taneepanichskul, 2015), aspectos que no se abordaron en el presente trabajo. Autores como Moreno-Tochihuitl, Gallegos-Cabriales, Salazar-González y Domínguez-González (2018) mencionan que el apoyo familiar también es un factor indispensable para mantener niveles óptimos de glucosa, puesto que la familia facilita al paciente apegarse a la dieta ya que es dentro de la familia donde se desarrollan hábitos, costumbres y valores que regulan las conductas individuales.

Otro aspecto que pudo intervenir es el estatus socioeconómico, basado en ingresos económicos, la educación y la ocupación, el cual ha demostrado, según Zhang et al. (2013), influir en el control glucémico, encontrando que los bajos ingresos económicos se asocian con mayores probabilidades de alteración en la glucosa, y en pacientes con DT2, la baja educación y la ocupación que no requiere trabajo fisico están relacionados con un control glucémico deficiente. Le religión tambien juega un papel importante en el control glucémico de las personas con DT2, ya que autores como Newlin, Melkus, Tappen, Chyun y Koenig (2008), demuestran que la religión y la espiritualidad se relacionaron directamente con el control glucémico, mientras que How, Ming y Chin (2011) encontraron que las personas con mayor religiosidad y que asistían a la iglesia tenían un mejor control glucémico, en este trabajo no se indago.

\section{Conclusiones}

Por lo anterior se concluye que la autodeterminación (motivación interna) es importante en la vida de las personas con DT2, influyendo en la dieta y el ejercicio físico, ya que la mayoría de la población se encontró autodeterminada tanto para llevar una dieta sana como para hacer ejercicio físico, pero ésta no influyó directamente en el control glucémico. Se sugiere seguir estudiando aspectos que puedan interferir en la autodeterminación y en el control glucémico, aspectos sociodemográficos como los años de diagnóstico, la edad, los ingresos económicos, la ocupación, comorbilidades como la depresión y el estrés, así como aspectos religiosos como la espiritualidad, los hábitos alimenticios por mencionar algunos., en futuros estudios con una muestra mayor que en la presente investigación.

\section{REFERENCIAS}

American Diabetes Association. (2018). Standards of medical care in diabetes-2018, Diabetes care, 41(1). Recuperado de: https://diabetesed.net/wp-content/uploads/2017/12/2018-ADAStandards-of-Care.pdf

Aspano, M., Lobato, S., Leyton, M., Batista, M., \& Jiménez, R. (2016). Predicción de la motivación en las etapas de cambio de ejercicio más activos. RETOS. Nuevas Tendencias en Educación Física, Deporte y Recreación, 30(1), 87-91.

Banco Mundial (2017). Nota descriptiva: La diabetes, una amenaza invisible para el desarrollo. Recuperado de: http://www.bancomundial.org/es/news/feature/2017/01/04/diabet es-invisible-threat-development

Consejo Nacional de Evaluación de la Política de Desarrollo Social (2015). Medición de la pobreza, Nuevo León, 2010- 2015. Indicadores de pobreza por municipio. Recuperado de: https://www.coneval.org.mx/coordinacion/entidades/NuevoLeon/ Paginas/pobreza municipal2015.asp

Deci, E., \& Ryan, R. (1985). Intrinsic motivation and self-determination in human behavior. New York: Plenum Press.

Encuesta Nacional de Salud y Nutrición de Medio Camino. (2016). Informe final de resultados. Recuperado de: http://www.promocion.salud.gob.mx/dgps/descargas1/doctos 201 6/ensanut mc 2016-310oct.pdf

Federación Internacional de Diabetes. (2017). Diabetes atlas de la FID, octava edición, 2017. Recuperado de: www.diabetesatlas.org

Fundación Mexicana para la Salud. (2015). Carga económica de la diabetes mellitus en México, 2013. México, D.F. Recuperado de: http://funsalud.org.mx/portal/wp-content/uploads/2015/08/CargaEconomica-Diabetes-en-Mexico-2013.pdf

Gourlan, M., \& Trouillound, D. (2015). Motivational Profiles for Physical Activity Practice in Adults with Type 2 Diabetes: A SelfDetermination Theory Perspective. Behavioral Medicine, 42(4), $227-$ 237. doi: $10.1080 / 08964289.2014 .1001810$

Halvari, H., Healey, J., Olafsen, A., Byrkjeland, R., Deci, E., \& Williams, G. (2016). Physical activity and motivational predictors of changes in health behavior and health among DM2 and CAD patients. Scandinavian journal of medicine y science in sports, 27(11), 14541469. doi:10.1111/sms.12757

How, C., Ming, K., \& Chin, C. (2011). Does religious affiliation influence glycaemic control in primary care patients with type 2 diabetes mellitus? Mental Health in Family Medicine, 8(1), 21- 28.

Instituto Nacional de Estadística y Geografía (2015). Características educativas de la población. Recuperado de: https://www.inegi.org.mx/temas/educacion/

Instituto Nacional de Salud Pública. (2013). Encuesta Nacional de Salud y Nutrición 2012. Resultados por entidad federativa. Nuevo León. Cuernavaca: México. Recuperado de: https://encuestas.insp.mx/ 
Kálcza-Jánosi, K., Williams, G., \& Szamosközi, I. (2017). Intercultural differences of motivation in patients with diabetes. A comparative study of motivation in patients with diabetes from Transylvania and USA. Transylvanian Journal of Psychology, 17(1), 3-17.

Koponen, A., Simonsen, N., \& Suominen, S. (2016). Determinants of physical activity among patients with type 2 diabetes: the role of perceived autonomy support, autonomous motivation and selfcare competence. Psychology, Health \& Medicine, 22(3), 332-344. doi:10.1080/13548506.2016.1154179

Mazzini, F., Ubilla, W., \& Moreira, T. (2017). Factores predisponentes que afectan la salud bucodental en pacientes con diabetes mellitus. Revista Odontológica Mexicana, 22(2), 103-108.

Moreno-Tochihuitl, M., Gallegos-Cabriales, E., Salazar-González, B., \& Domínguez-González, E. (2018). Teoría de rango medio: procesos familiares que predicen el descontrol glucémico en diabetes mellitus 2. Journal Health NPEPS, 3(2), 634-648. doi:10.30681/252610102929

Newlin, K., Melkus, G., Tappen, R., Chyun, D., \& Koenig, H. (2008). Relationships of Religion and Spirituality to Glycemic Control in Black Women With Type 2 Diabetes. Nursing Research, 57(5), 331339.

Organización Mundial de la Salud. (2016). Informe mundial sobre la diabetes. Geneva: Switzerland. Recuperado de: http://apps.who.int/iris/bitstream/10665/254649/1/978924356525 5-spa.pdf

Saengtipbovorn, S., \& Taneepanichskul, S. (2015). Effectiveness of Lifestyle Change Plus Dental Care Program in Improving Glycemic and Periodontal Status in Aging Patients with Diabetes: A Cluster, Randomized, Controlled Trial. Journal of Periodontology, 8(4), 507515. doi:10.1902/jop.2015.140563

Sánchez-Cruz, J., Hipólito-Lóenzo, A., Mugártegui-Sánchez, S., \& YáñezGonzález, R. (2016). Estrés y depresión asociados a la no adherencia al tratamiento en pacientes con Diabetes Mellitus tipo 2. Atención Familiar, 23(2), 43- 47.

Sanz, M., Ceriello, A., Buysschaert, M., Chapple, I., Demmer, R., Graziani, F., Herrera, D., Jepsen, S., Lione, L., Madianos, P., \& Mathur, M. (2017). Scientific evidence on the links between periodontal diseases and diabetes: Consensus report and guidelines of the joint workshop on periodontal diseases and diabetes by the International Diabetes Federation and the European Federation of Periodontology. Journal of Clinical Periodontology, 45(2), 138-149. doi:10.1111/jcpe.12808

Secretaría de Salud. (1987). Reglamento de la Ley General de Salud en materia de Investigación para la Salud. México, D.F. Recuperado de: http://www.diputados.gob.mx/LeyesBiblio/regley/Reg_LGS_MIS.pd f

Shahar, et al. (2016). Family Support and Self-Motivation Influence Dietary Compliance and Glycaemic Control among Type 2 Diabetes Mellitus Outpatients. Jurnal Sains Kesihatan Malaysia, 14(2), 39- 47. doi:10.17576/JSKM-2016-1402-05

Ternois, M. (2017). The oral cavity: A mirror of diabetes. Presse Medicale, 46(9), 822- 830. doi:10.1016/j.lpm.2017.04.001
Trief, P., Cibula, D., Delahanty, L., \& Weinstock, R. (2016). Selfdetermination theory and weight loss in a Diabetes Prevention Program translation trial. Journal of Behavioral Medicine, 40, 483-493. doi:10.1007/s10865-016-9816-9

Williams, G. C., Freedman, Z. R., \& Deci, E. L. (1998). Supporting autonomy to motivate glucose control in patients with diabetes. Diabetes Care, $21,1644-1651$

Zhang, H., Xu, W., Dahl, A., Xu, Z., Wang, H., \& Qi, X. (2013). Relation of socio-economic status to impaired fasting glucose and Type 2 diabetes: findings based on a large population-based crosssectional study in Tianjin, China. Diabetic Medicine, 30(1),157- 162. 